\title{
Brachial and Lumbosacral Plexus and Peripheral Nerves
}

\author{
Roberto Gasparotti and Lubdha Shah
}

\section{Learning Objectives}

- Review the anatomy of the peripheral nerves and brachial and lumbosacral plexi.

- Understand the utility of different magnetic resonance imaging (MRI) sequences and magnetic resonance neurography (MRN) for identifying peripheral nerve diseases.

- Describe the imaging characteristics of different peripheral nerve diseases to help narrow the differential diagnosis.

- Discuss the role of advanced imaging techniques.

\subsection{Introduction}

The diagnostic work-up of peripheral neuropathies relies on the patient's clinical history, physical examination, and electrophysiological studies, which usually provide enough information about the location, severity, and the etiology of the underlying nerve injury in the majority of patients. However, electrodiagnostic studies, such as electromyography (EMG), do not display the anatomic detail needed for precise localization and treatment planning because of the deep location of nerves and the variable innervation of regional muscles. EMG sensitivity for lumbosacral radiculopathy ranges from 49 to $86 \%$. Therefore, imaging particularly with magnetic resonance imaging (MRI)/magnetic resonance neurography (MRN) and ultrasound (US) has an increasing role in the evaluation of peripheral

R. Gasparotti

U.O. Neuroradiologia, ASST Spedali Civili, Università degli Studi di Brescia, Brescia, Italy

e-mail: roberto.gasparotti@unibs.it

L. Shah $(\varangle)$

Department of Radiology, University of Utah Health Sciences

Center, Salt Lake City, UT, USA

e-mail: lubdha.shah@hsc.utah.edu neuropathies [1]. Although US is more operator-dependent than MRI and less effective in cases of deep nerves of the pelvis and lumbosacral plexus, it is very effective for dynamic assessment of superficial peripheral nerves abnormalities, such as changes in nerve caliber, continuity, and echogenicity [2].

\subsection{Anatomy of Peripheral Nerves}

Peripheral nerves range from 1 to $20 \mathrm{~mm}$ in size, the largest being the sciatic nerve. Peripheral nerves are formed by multiple axons, grouped into fascicles, the number of which depends on the size and length of the nerve. Endoneurium invests the Schwann cell-axon complex, with the inner border represented by the Schwann cell basement membrane and its outer border by the perineurium. The epineurium, the outermost connective tissue sheath, envelops the nerve and provides mechanical support for the axons. Endoneurial fluid within each fascicle is isolated from the general extracellular space by tightly adherent epithelial-like cells of the perineurium and from the circulating blood by the tight junctions between the endothelial cells of the endoneurial capillaries. The endoneurium and perineurium form a functional, relatively impermeable barrier known as the blood-nerve interface that protects the peripheral nervous system against toxic and infectious agents.

\subsection{Brachial Plexus (BP)}

The ventral rami of the $\mathrm{C} 5$ through $\mathrm{T} 1$ nerves give rise to the BP. The BP structures travel through the supraclavicular fossa paralleling the subclavian artery. Individual ventral rami or roots are best seen on axial and coronal images. The sagittal plane is helpful for following the BP structures from the spine to the axilla as they lie perpendicular to the plane. The upper (C5-C6), middle (C7), and lower (C8-T1) trunks travel between anterior and middle scalene muscles, forming the lateral border of the scalene triangle [3]. In the 
supraclavicular triangle, the trunks divide into anterior and posterior divisions just before passing posterior to the clavicle and going from the lateral aspect of the anterior scalene muscle to the lateral border of the first rib [3]. The cords (lateral, medial, and posterior) course from the mid-clavicle to the inferomedial coracoid process and are named according to location relative to the axillary artery. At the pectoralis minor muscle, just medial to the coracoid, axillary vein, and axillary artery, the cords change into the five terminal branches (radial, axillary, musculocutaneous, median, and ulnar nerves).

\subsection{Lumbosacral Plexus (LSP)}

The ventral rami of the L1-L4 spinal nerve roots and a small contribution from the 12 th thoracic nerve coalesce within or posterior to the psoas major muscle to form the lumbar plexus [2]. The lumbar plexus gives rise to the iliohypogastric (L1), ilioinguinal (L1), genitofemoral (L1-L2), femoral (L2-L4), and lateral femoral cutaneous (L2-L3) nerves, which emerge lateral to the psoas major muscle. The obturator nerve and lumbosacral trunk come forward from the medial border of the psoas muscle. A minor (peroneal) branch of L4 combines with the ventral ramus of L5 to form the lumbosacral trunk. The latter descends over the sacral ala and joins the S1 to S3 ventral rami on the anterior aspect of the piriformis muscle to form the sacral plexus [4]. The sacral plexus gives rise to the sciatic (L4-S3), pudendal (S2-S4), superior gluteal (L4-S1), and inferior gluteal (L5-S2) nerves.

\subsection{Magnetic Resonance Neurography (MRN)}

Although conventional MRI can provide structural information, it is limited in its ability to visualize peripheral nerves due to low contrast resolution between the small nerves, muscles, and vessels, signal intensity variability, and pulsatility artifacts. These disadvantages have largely been overcome with MRN. The MRN technique relies on high magnetic field strength, T2-weighted sequences with fat suppression, dedicated surface coils, and small field of view (FOV). MRN can identify specific characteristics of nerve morphology, such as internal fascicular pattern, longitudinal variations in signal intensity and caliber, and connections and relations to other nerves or plexuses [5]. The most efficient fat suppression is obtained with T2-weighted short time inversion recovery (STIR) sequences, which selectively eliminate the fat signal using an inversion recovery pulse of $150 \mathrm{~ms}$. These sequences, however, are characterized by poor signalto-noise ratio and pulsatility artifacts caused by vessels. Alternative methods for fat suppression are T2 spectral adia- batic inversion recovery imaging (SPAIR) or DIXON-type fat suppression, which both have improved signal-to-noise ratio, at the expense of lower contrast resolution. In order to obtain the best compromise between spatial resolution, FOV, and acquisition time, MRN sequences should be adapted to the anatomical region and the best echo time should be carefully selected in order to obtain a satisfactory differentiation between nerves and muscles, as the signal intensity of the nerve is very sensitive to small changes (Fig. 20.1).

Three-dimensional (3D) MRN is a refinement of conventional MRN. It is typically acquired with isotropic voxels, with the advantage of generating oblique and curved-planar reformations of nerve roots, peripheral nerves, and plexuses. This feature is particularly useful for imaging brachial and lumbosacral plexi, which cannot be fully displayed by $2 \mathrm{D}$ imaging (Fig. 20.2). On MRN axial images, normal nerves are rounded or ovoid structures, typically isointense to slightly hyperintense on $\mathrm{T} 2$, depending on the size of the nerve, on the amount of endoneurial fluid, and the degree of fat suppression. On T1 images, nerves are isointense to the adjacent muscles. The epineurium appears as a thin hypointense rim, and the transverse and longitudinal fascicular pattern may be identified in larger nerves such as the sciatic nerve. Diseased nerves become $\mathrm{T} 2$ hyperintense to muscle and are focally or globally enlarged [6]. The signal intensity change is due to increased water content in the epineurial space caused by blood-nerve barrier damage, axoplasmic flow blockade, inflammation, distal Wallerian degeneration and is relatively independent from

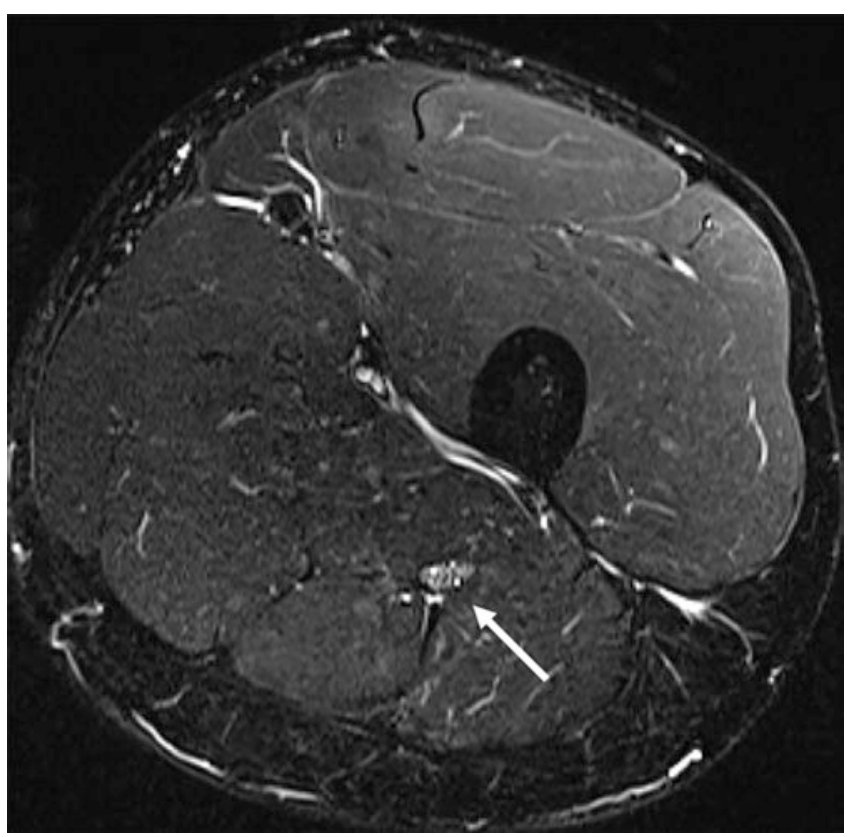

Fig. 20.1 MRN (3 T), axial T2-STIR section at mid-thigh. Normal subject. The right sciatic nerve (arrow) is moderately hyperintense compared to the adjacent muscles and its transverse fascicular pattern is clearly identifiable 


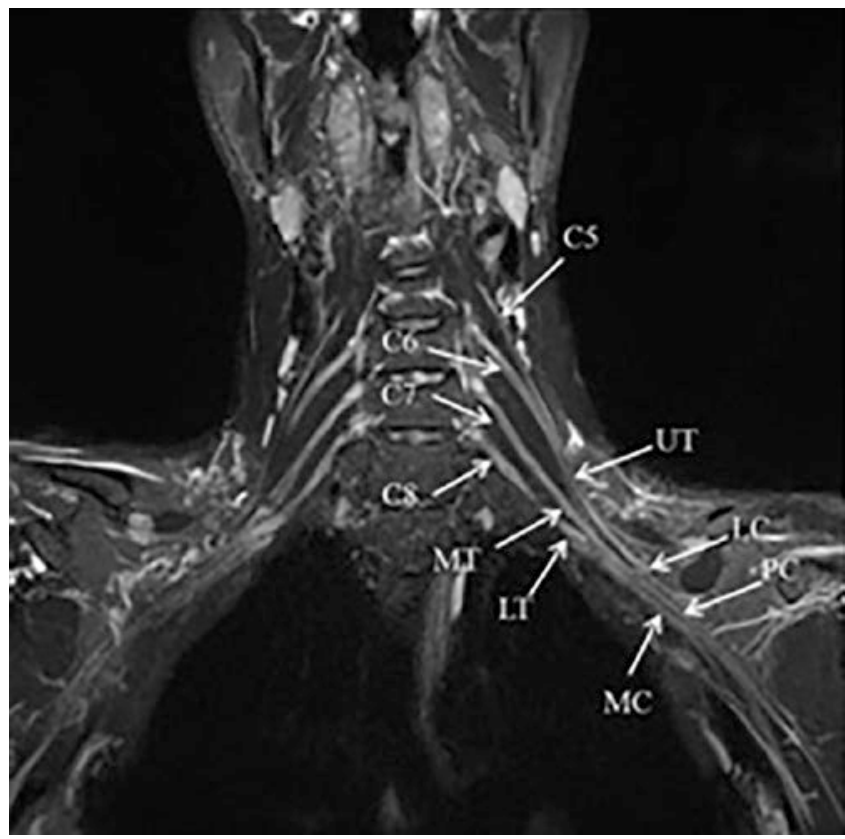

Fig. 20.2 3D MRN (3 T) of the brachial plexus. Oblique coronal reformat. The supra and infraclavicular segments of the brachial plexus are simultaneously displayed in a single image. Upper trunk (UT), Middle trunk (MT), Lower trunk (LT), Lateral cord (LC), Posterior cord PC), Medial cord (MC)

the etiology of the neuropathy. Neuropathies with different etiologies cannot be distinguished only on the basis of signal intensity changes as there are no reliable quantitative methods to differentiate between the signal intensities of normal versus abnormal nerves. MRN has the advantage of a simultaneous exploration of nerves and muscles. Importantly, muscle denervation represents a useful MR sign of peripheral nerve disease. In the acute phase of muscle denervation, increased signal intensity can be observed in T2 sequences as early as $24 \mathrm{~h}$ after nerve injury and lasting for more than 2 months [7]. In the subacute phase, a gradual decrease of $\mathrm{T} 2$ signal intensity is associated with fatty replacement, which progresses to fatty atrophy of the affected muscles, best displayed by T1 images. The MR changes precede the earliest EMG findings of denervation, which are not detectable until the second week.

A comprehensive MRI protocol for the investigation of peripheral nerves should include MRN, which provide both structural and functional information on the nerves and muscle denervation, $\mathrm{T} 1$ sequences which are helpful for a precise anatomical identification of nerves and for the identification of muscular atrophy, and contrast-enhanced T1 sequences for the evaluation of the blood-nerve barrier integrity. MRN is effective in the diagnostic work-up of traumatic nerve injuries [8], nerve entrapment syndromes [9], and nerve tumors [10]. Lumbosacral MRN may demonstrate abnormal intraneural $\mathrm{T} 2$ signal in a substantial portion of patients with clinical symptoms of lower extremity radiculopathy and correlates with findings of active radiculopathy on EMG [11]. Recently MRN has been proposed for the evaluation of hereditary and immune-mediated disorders of peripheral nerves [12].

\subsection{Diffusion Tensor Imaging}

Diffusion tensor imaging (DTI) is a novel technique which has been recently applied to the investigation of peripheral nerve disorders. DTI provides "microstructural" information about nerve integrity. Nerves are characterized by greater water diffusion anisotropy compared to the surrounding tissues, due to a barrier to water diffusion formed by axonal myelin sheaths. Diffusion imaging of the peripheral nerves is challenging because of their small size and course, particularly the BP because of the geometric distortion and artifacts along the course of the nerves between the neck and the shoulder. Additionally, DTI gives quantitative information about the degree and direction of water diffusion: fractional anisotropy (FA), apparent diffusion coefficient (ADC), mean diffusivity (MD), axial diffusivity (AD), and radial diffusivity (RD) [13]. Reconstructing the diffusion information to characterize the integrity of the white matter tracts (diffusion tensor tractography, DTT) yields 3D representations of anisotropic nerve fibers. Successful tracking of the major peripheral nerves can be obtained using the same approach as deterministic tractography of the brain white matter bundles. DTI has been extensively applied to the median nerve at the carpal tunnel and more recently to the BP and LSP, although its overall diagnostic value in clinical routine is still to be ascertained. Although DTI and tractography are not widely used clinically, good reproducibility of DTI quantitative analysis with only small variations in calculated FA and $\mathrm{ADC}$ in the BP of normal volunteers has been shown [14].

\section{Key Point}

- MRN can characterize nerve morphology, longitudinal variations in signal intensity and caliber, and connections and relations to other nerves or plexuses to help identify pathology in correlation with the patient's clinical history, physical examination, and electrophysiological studies.

\subsection{Traumatic Injuries of Peripheral Nerves}

Peripheral nerve injuries are classified into three types: neuropraxia, axonotmesis, and neurotmesis, which are defined by the histopathology and specific anatomic structures of the nerve unit that are damaged (Table 20.1). Neuropraxia 
Table 20.1 MR imaging in peripheral nerve injuries according to Seddon's classification

\begin{tabular}{|l|l|}
\hline Classification & MR \\
\hline Neurapraxia & $-\begin{array}{l}\text { T2 hyperintensity of the nerve (within } 24 \mathrm{~h} \text { of } \\
\text { trauma) } \\
-\end{array}$ \\
\hline Axonotmesis & $-\begin{array}{l}\text { To muscle denervation } \\
\text { with fascicular hypertrophy, with or without } \\
\text { neuroma in continuity }\end{array}$ \\
& - Muscle denervation
\end{tabular}

is the mildest form of traumatic plexopathy, in which there is temporary loss of function, near complete recovery, and the absence of denervation atrophy of the muscle. The nerve is grossly intact but functionally impaired with slowed or absent conduction in the involved segment. More serious injury to the neural axons results in axonotmesis and neurotmesis. In axonotmesis, there is loss of axonal continuity and the endoneurium, with Wallerian degeneration distal to the site of injury. As such, recovery requires nerve regeneration to regain function. Depending on the severity of injury, MRN will demonstrate varying degrees of nerve enlargement, disruption, and effacement of nerve fascicles. In neurotmesis, disruption of the axons, myelin sheath, and surrounding perineurium and epineurial layers typically requires surgical intervention. MRN may reveal a neuroma in continuity or complete transection of the nerve with formation of an end-bulb neuroma [15]. EMG and nerve conduction studies provide functional information but are unable to differentiate axonotmesis from neurotmesis. MRI can be used to differentiate neurapraxia from axonotmesis and neurotmesis through combined assessment of the characteristics of the injured nerve, typically represented by: (a) nerve enlargement with longitudinal variations in size, (b) increase in signal intensity, (c) signal intensity anomalies of the corresponding muscles, reflecting acute or subacute denervation.

\subsection{Traumatic Injuries of the Brachial Plexus}

MR imaging (including MR myelography and MRN) is primarily used to locate the injury level, i.e., the distinction between preganglionic lesions (avulsion of the cervical nerve roots from the spinal cord) and postganglionic lesions (distal to the dorsal root ganglion), which is important for prognosis and therapeutic management [16] (Table 20.2). Multiplanar and curved reformats from 3D MRN are helpful to identify postganglionic lesions. Of note, MRI within 1 month of injury can be challenging to evaluate because hemorrhage and edema can compromise visualization of the thecal sac and nerves. In adults, the majority of brachial plexopathies
Table 20.2 MR imaging findings in different peripheral nerve injuries on MR Myelography

\begin{tabular}{|l|l|}
\hline Brachial plexus injuries & MR \\
\hline Partial avulsion & $\begin{array}{l}\text { Traumatic pseudomeningoceles } \\
\text { Ventral and dorsal roots not identifiable } \\
\text { Denervation edema of the posterior } \\
\text { paraspinal muscles }\end{array}$ \\
\hline $\begin{array}{l}\text { Reduced number of rootlets on MIP } \\
\text { projections } \\
\text { Ventral or dorsal root absent on axial } \\
\text { sections with mild abnormalities of dural } \\
\text { sleeves }\end{array}$ \\
\hline $\begin{array}{l}\text { Pwelling and increased signal intensity of } \\
\text { the roots, trunks and cords of the brachial } \\
\text { plexus } \\
\text { Tortuosity and increased intensity of the } \\
\text { infraclavicular brachial plexus } \\
\text { Post-traumatic neuromas along the course } \\
\text { of the roots and/or primary trunks }\end{array}$
\end{tabular}

are due to traumatic injuries, usually resulting from highvelocity trauma, such as motorcycle accidents. In the pediatric population, traumatic brachial plexopathy is often secondary to excessive severe traction force exerted on the brachial plexus during complicated deliveries. In preganglionic injuries, MRI may reveal spinal cord edema in the acute phase (T2 hyperintensity) [17]. There may be susceptibility effects from hemosiderin, accentuated on $\mathrm{T} 2 *$ gradient susceptibility images. A helpful clue to preganglionic avulsion injury is the formation of a pseudomeningocele, which is seen in $80 \%$ of traumatic avulsions; notably, pseudomeningoceles take time to develop. This lesion is a sequela of traction on the cervical roots and meninges resulting in leakage of cerebrospinal fluid in contiguous areas, anterior epidural space, neural foramina, perivertebral spaces, surrounded by a thin membrane. In complete avulsions, the ventral and dorsal roots are not identifiable, whereas partial avulsions are characterized by rarefaction of the ventral or dorsal rootlets, with mild abnormalities of the dural sleeves (Fig. 20.3). Changes of muscular denervation, including alterations in signal intensities and contrast enhancement, in the posterior cervical paraspinal muscles, particularly in the multifidus muscle, are an indirect imaging finding of a preganglionic injury.

In postganglionic injuries, the nerve may be stretched and remain in continuity. The acutely injured nerve is thickened and typically $\mathrm{T} 2$ hyperintense. The secondary trunks are often characterized by marked tortuosity. Post-traumatic neuromas can be identified along the course of the roots or primary trunks as neuromas "in continuity," characterized by fusiform swelling, or as "stump" neuromas (Fig. 20.4). DTT has been proposed as a diagnostic tool to assess traumatic BP lesions, due to high diagnostic accuracy in the identification of radicular avulsions, especially when extensive dural scar or CSF artifacts limit the usefulness of MRI [18] (Fig. 20.5).

For traumatic injuries of the upper limb, US is the examination of choice and can be performed imme- 

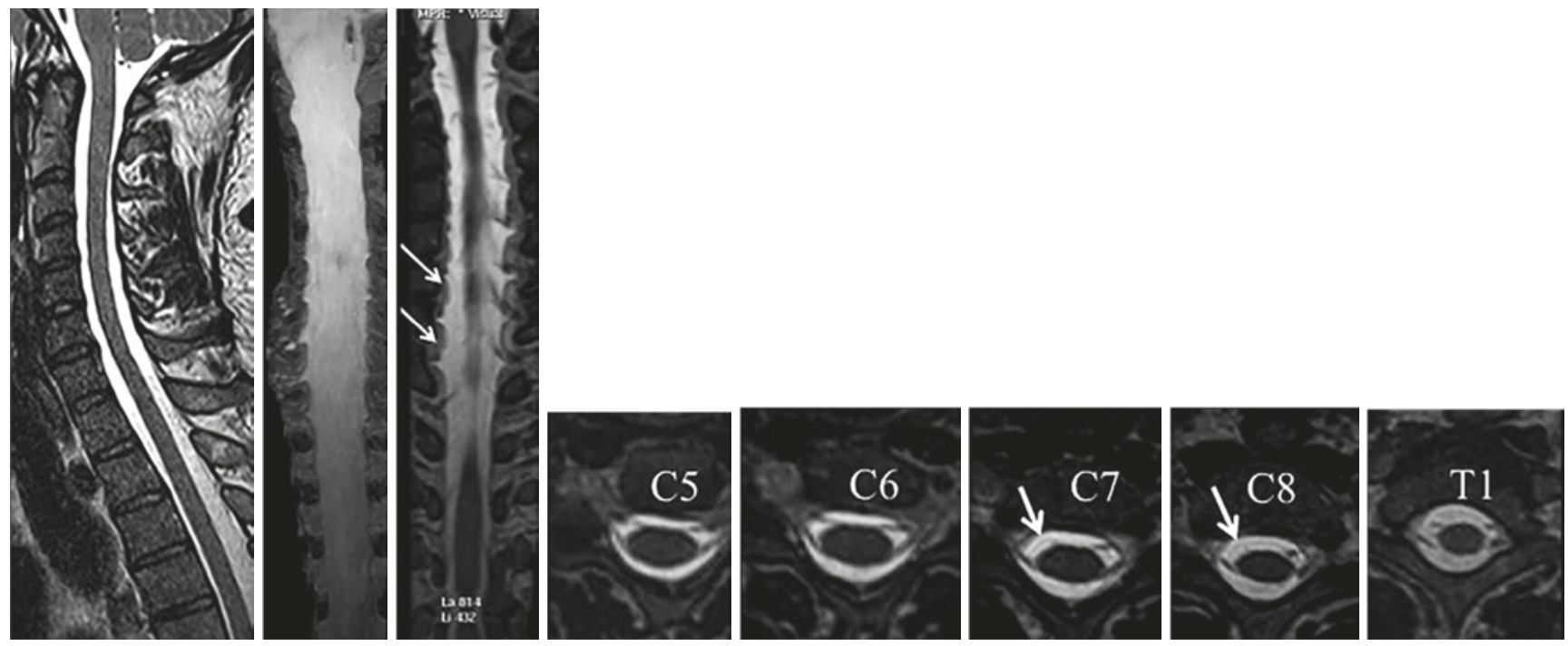

Fig. 20.3 22-year-old male, motor cycle accident, with complete right brachial plexus palsy. 3D MR Myelography. Right C7 and C8 (partial) avulsions of the ventral nerve roots (arrows)

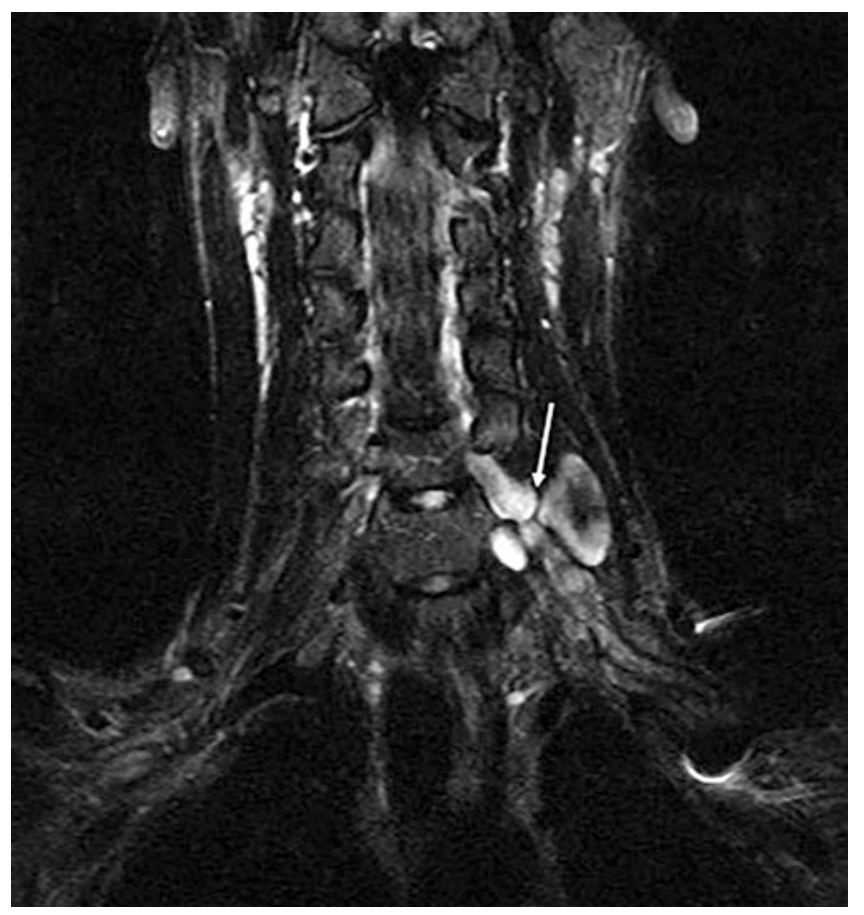

Fig. 20.4 MRN, traumatic injury of the left brachial plexus. Neurotmesis of the left C6 nerve root: stump neuroma (arrow)

diately after trauma to demonstrate neurotmesis-type lesions to show nerve discontinuity. MRN can identify axonotmesis-type lesions, which appear as global nerve enlargement with mild fascicular hypertrophy that extends longitudinally and requires longer time for nerve regeneration.

DTI can be used to assess the nerve regeneration after surgery [19].

\subsection{Traumatic Injuries of the Lumbosacral Plexus, Sciatic and Femoral Nerves}

Indirect injury to the LSP may occur as a part of lumbar spine injuries, pelvic fractures, and hip fractures or dislocations. Pelvic or hip injuries typically cause stretch- or tractionrelated partial plexopathy and, less commonly, nerve avulsions. The avulsions of lumbosacral nerve roots typically involve L4, L5, and S1. MRN can locate the level of the injury and provide information about the associated muscle denervation (Fig. 20.6). Fibular nerve can be injured in fibular fractures and knee dislocations as well as in habitual leg crossing or repetitive exercise in athletes.

\section{Key Point}

- A pseudomeningocele is a preganglionic injury that is seen in $80 \%$ of traumatic avulsions. It results from traction on the cervical roots and meninges with CSF leakage into contiguous areas.

Iatrogenic injury to the LSP may result from surgical gynecologic or anesthetic procedures, compression, traction, and vascular insults [20]. Traumatic injuries of the sciatic nerve can also occur as a complication of prosthetic hip surgery, most frequently involving the fibular division. Injuries of the femoral nerve can be secondary to pelvic trauma or iatrogenic in nature with nerve compression from hematoma or pseudoaneurysm during femoral artery puncture, respectively. The intrapelvic femoral nerve may show increased T2 signal and size or deviation by hematoma, with denervation of the iliopsoas muscle. 

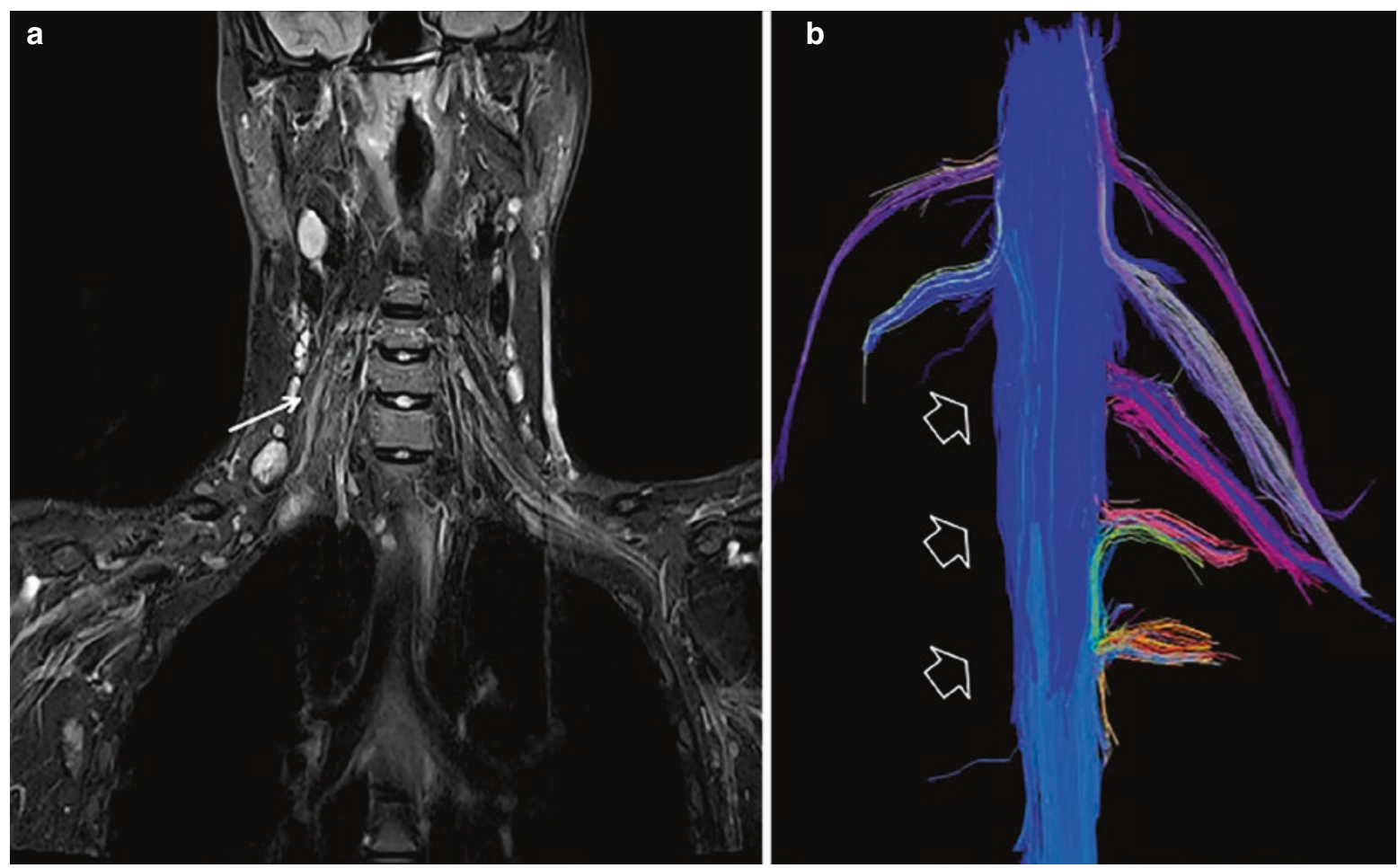

Fig. 20.5 Traumatic injury of the right brachial plexus. (a) MRN: the right brachial plexus is barely identifiable (arrow). (b) Tractography: right $\mathrm{C} 7, \mathrm{C} 8, \mathrm{~T} 1$ nerve root avulsions (open arrows)
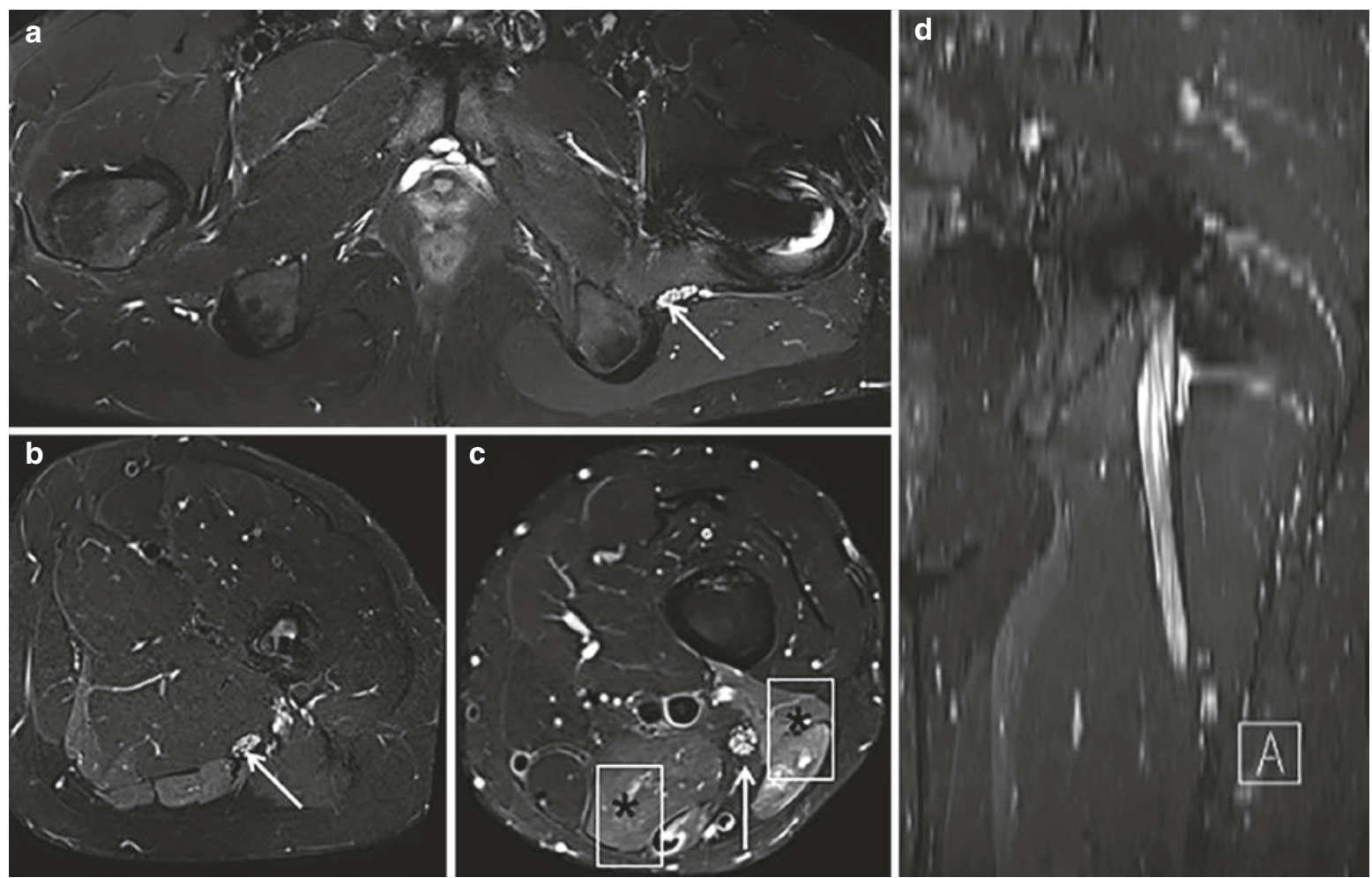

Fig. 20.6 24-year-old male, road accident, with left femoral shaft fracture, sciatic nerve injury. MRN (1.5 T), axial T2-STIR at pelvic floor acterized by fascicular hypertrophy (arrows). Increased signal intensity of semimembranosus, semitendinosus, and long head of the biceps (a), proximal and mid-thigh $(\mathbf{b}, \mathbf{c})$, sagittal oblique reformat (d). femuri, due to acute denervation Enlargement and hyperintensity of the left sciatic nerve, which is char- 
Although nerve abnormalities in the thigh can be difficult to detect, denervation of the quadriceps femoris may be a clue to femoral nerve injury distal to the groin ligament.

\subsection{Entrapment Neuropathies}

Peripheral nerve compression or entrapment usually occurs at specific anatomic locations, often close to limb joints, such as sites where a nerve courses through fibro-osseous or fibromuscular tunnels or penetrates muscles (Table 20.3). US and MRN can provide direct anatomic visualization of the entrapped nerves. US has the advantage of providing dynamic evaluation of nerve entrapment. MRN demonstrates varying degrees of signal intensity within the nerve, proximal to the site of entrapment, and the associated muscle denervation changes. Proximal nerve enlargement and fascicular hypertrophy and distal flattening can develop with increasing severity of neuropathy.

Table 20.3 Common nerve entrapment syndromes

\begin{tabular}{|c|c|c|}
\hline $\begin{array}{l}\text { Nerve entrapment } \\
\text { syndrome }\end{array}$ & $\begin{array}{l}\text { Involved } \\
\text { nerve }\end{array}$ & Site of entrapment \\
\hline $\begin{array}{l}\text { Carpal tunnel } \\
\text { syndrome }\end{array}$ & $\begin{array}{l}\text { Median } \\
\text { nerve }\end{array}$ & $\begin{array}{l}\text { Carpal tunnel, at the level of } \\
\text { hamate (proximal alterations at } \\
\text { the level of pisiform) }\end{array}$ \\
\hline Ulnar neuropathy & $\begin{array}{l}\text { Ulnar } \\
\text { nerve }\end{array}$ & Cubital tunnel \\
\hline $\begin{array}{l}\text { Fibular nerve } \\
\text { entrapment }\end{array}$ & $\begin{array}{l}\text { Fibular } \\
\text { nerve }\end{array}$ & $\begin{array}{l}\text { At fibular head or deep to the } \\
\text { origin of peroneus longus muscle }\end{array}$ \\
\hline $\begin{array}{l}\text { Piriformis } \\
\text { syndrome }\end{array}$ & $\begin{array}{l}\text { Sciatic } \\
\text { nerve }\end{array}$ & Sciatic notch \\
\hline $\begin{array}{l}\text { Tibial nerve } \\
\text { entrapment }\end{array}$ & $\begin{array}{l}\text { Tibial } \\
\text { nerve }\end{array}$ & Tarsal tunnel \\
\hline
\end{tabular}

\subsection{Carpal Tunnel Syndrome}

Carpal tunnel syndrome (CTS) is the most common peripheral nerve entrapment syndrome and has various etiologies, including repetitive trauma, metabolic and hormonal conditions, and ganglion cysts. While US has $77.6 \%$ sensitivity and $86.8 \%$ specificity for diagnosing CTS, sensitivity of MRN is above 90\%. On MRN, abnormal nerve signal length and cross-sectional area at the distal radioulnar joint are the best predictors of the severity [21]. Correlation between median nerve DTI metrics in the carpal tunnel and electrophysiology has been recently reported [22]. MRN is particularly useful for the evaluation of atypical CTS, when assessing for space-occupying lesion, and in patients with persistent symptoms after carpal tunnel release.

\subsection{Ulnar Neuropathy at the Elbow}

Ulnar nerve entrapment at the cubital tunnel is the second most common entrapment neuropathy. The dynamic US evaluation during flexion/extension of the elbow is crucial for showing subluxation of the ulnar nerve. MRN can discriminate between symptomatic ulnar nerve entrapment at the elbow and asymptomatic controls with high diagnostic accuracy. In patients with electrophysiologically and clinically evident ulnar nerve entrapment, DTI may show regional FA decrease at the cubital tunnel, correlating with symptom severity (Fig. 20.7).

\section{Key Point}

- Although both US and MRN offer anatomic delineation of entrapped nerves, US has the advantage of providing dynamic evaluation of nerve entrapment.
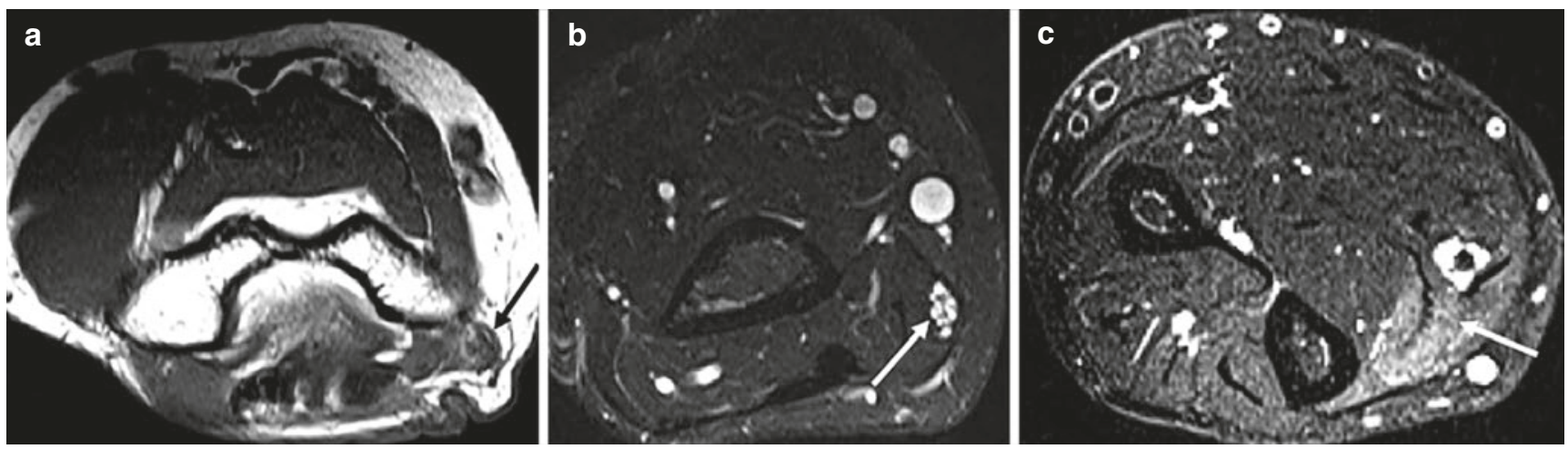

Fig. 20.7 48-year old male with acute onset of right forearm pain and paresthesia. Ulnar nerve entrapment at the cubital tunnel. (a) Axial T1 image at the right elbow (a), MRN at distal arm (b), and forearm (c). Deformation of the right ulnar nerve (black arrow in a). Proximal to the entrapment site, the ulnar nerve is enlarged and hyperintense with fascicular hypertrophy (arrow in b). Denervation edema of the flexor carpi ulnaris (arrow in c) 


\subsection{Non-traumatic Brachial and Lumbosacral Plexopathies}

Idiopathic brachial plexitis or idiopathic neuralgic amyotrophy [INA] ( $a k a$ Parsonage Turner syndrome) is a self-limiting inflammatory disorder usually affecting the unilateral BP, more commonly in males. Acute symptoms include neck, shoulder, and scapula pain. Muscle weakness ensues days or weeks after the initial pain, and the symptoms may last several months up to 3 years. MRN will show diffuse T2 hyperintensity and enlargement of affected neural structures, commonly C5 and C6 nerve roots and the upper trunk, and denervation edema in various muscles that does not follow a typical innervation pattern (e.g., isolated edema in the teres minor muscle).

Although an autoimmune/inflammatory etiology has been hypothesized, a recent alternative theory is fascicular torsion which more frequently occurs in the suprascapular and radial nerves. MRN will reveal the characteristic "bulls' eye sign" of central hypointensity surrounded by a hyperintense rim [23] (Fig. 20.8). MRN is also helpful in distinguishing INA from cervical spondylotic radiculopathy by demonstrating enlargement and increased signal intensity of cervical nerve roots at the level of foraminal stenosis, which correlate with the distribution of radicular pain and muscle weakness.

Idiopathic lumbosacral plexopathy (ILSP) is a self-limiting condition that presents with (sub)acute, severe, asymmetri- cal leg pain, followed by asymmetrical multifocal weakness and atrophy in the subsequent weeks or months. Though some studies have hypothesized an immune-mediated etiology, biopsies of distal cutaneous nerve segments have shown features of an inflammatory microvasculitis causing ischemic damage of the nerves [24]. Diabetic LSP neuropathy has similar clinical and pathological findings, suggesting that inflammation may form part of the final common pathway in both conditions. MRN shows increased signal intensity and mild contrast enhancement asymmetrically involving multiple nerve roots and terminal branches of the LSP as well muscle denervation changes.

LSP may be involved in retroperitoneal disorders, such as psoas abscess, hematoma, retroperitoneal fibrosis, and malignant disease (soft tissue or osseous), either by extrinsic compression or infiltration. Inflammatory sacroiliac joint arthritis may also involve the sacral component of the LSP.

Radiation plexopathy may manifest from a few months to years after brachytherapy or intraoperative radiation therapy, usually with doses exceeding $6000 \mathrm{cGy}$. This type of plexopathy is often painless and progresses slowly, in contradistinction to severely painful tumor-related plexopathy. Expected radiation-induced changes on MRN are uniform, symmetric thickening, T2 hyperintensity, and faint to no contrast enhancement of BP and LSP within the irradiated region. It may be difficult to separate the neural structures from each other. Recurrent tumor often shows a focal or diffuse het-
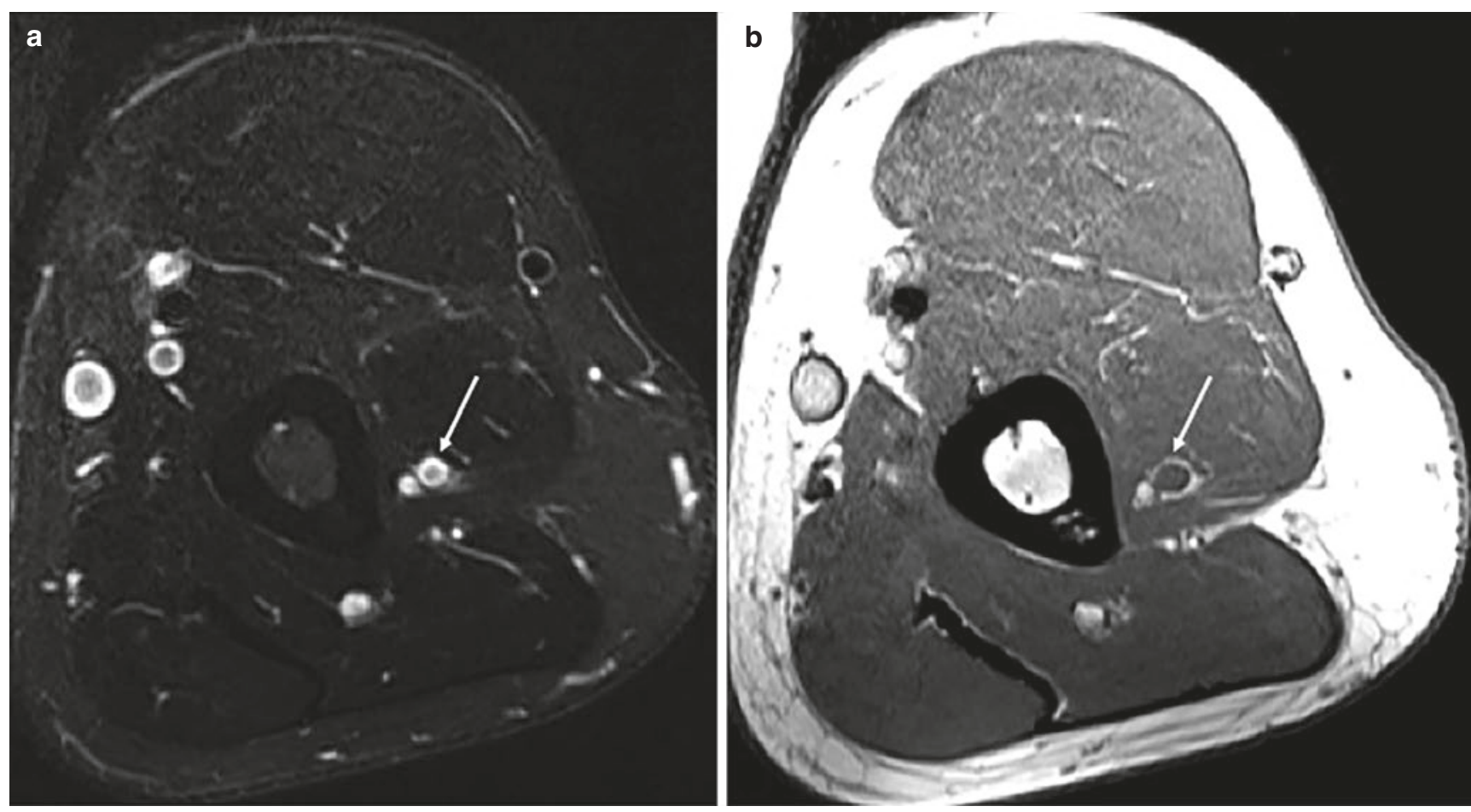

Fig. 20.8 62-year-old male with acute idiopathic neuralgic amyotrophy. (a) Axial T2 STIR, (b) axial T1. Swelling and increased signal intensity of the left radial nerve at mid-arm, with the bull's eye sign (arrow) 
erogeneously enhancing mass [25]. Follow-up imaging is helpful to differentiate radiation-induced inflammation from tumor recurrence as the former will show fibrotic changes over time.

\subsection{Thoracic Outlet Syndrome (TOS)}

TOS includes three disorders involving the neurovascular bundle in its course through the interscalene triangle and costoclavicular space: classic TOS (or neurogenic TOS), vascular TOS, and non-specific TOS. Classic TOS is the most frequent form, affecting middle-aged adults, especially women. It is characterized by chronic neck pain radiating to the supraclavicular region and arm, exacerbated by arm elevation, sometimes associated with paresthesias and numbness in C8/T1 dermatomes. Electrophysiological and clinical provocative tests are non-specific; however, MRI can reveal static or dynamic compression of the BP by structural abnormalities such as cervical or anomalous first rib, long C7 transverse process, muscular anomalies, or narrowed costoclavicular space [26].

\subsection{Immune-Mediated Neuropathies} (Table 20.4)

Immune-mediated neuropathies are diagnosed clinically according to the distribution of deficits and based on electrophysiology examination findings, laboratory parameters, and clinical context.

Guillain-Barre syndrome (GBS) or acute inflammatory demyelinating polyneuropathy (AIDP) is an acute syndrome, typically involving many nerves in a distal-to-proximal ascending distribution pattern. GBS is most commonly seen in the post respiratory or gastrointestinal tract infection or post-vaccination setting. MRI studies are usually not necessary for diagnosis but may be needed to exclude "mimic disorders." Initially, breakdown of the blood-nerve barrier is seen as intrathecal nerve root enhancement on MRI, particularly of the anterior spinal nerve roots [27]. Chronic inflam-

Table 20.4 MRI findings in inflammatory neuropathies

\begin{tabular}{|c|c|c|}
\hline & MRI & Distribution \\
\hline AIDP & Enhancement of spinal nerve roots & $\begin{array}{l}\text { Bilateral and } \\
\text { symmetrical }\end{array}$ \\
\hline CIDP & $\begin{array}{l}\text { Hypertrophy and T2 hyperintensity } \\
\text { of BP and LSP nerve roots, with } \\
\text { gradual normalization in distal } \\
\text { nerves }\end{array}$ & $\begin{array}{l}\text { Bilateral and } \\
\text { symmetrical }\end{array}$ \\
\hline MADSAM & $\begin{array}{l}\text { Hypertrophy and } \mathrm{T} 2 \text { hyperintensity } \\
\text { of peripheral nerve trunks }\end{array}$ & $\begin{array}{l}\text { Multifocal and } \\
\text { asymmetrical }\end{array}$ \\
\hline MMN & $\begin{array}{l}\text { Hypertrophy and } \mathrm{T} 2 \text { hyperintensity } \\
\text { Contrast enhancement of BP }\end{array}$ & Asymmetrical \\
\hline
\end{tabular}

matory demyelinating polyneuropathy (CIDP) is an acquired chronic, immune-mediated, multifocal, demyelinating neuropathy. It is characterized by symmetrical proximal and distal weakness, with sensory loss, impaired balance, and areflexia and affects primarily the spinal nerves, BP and LSP plexi, and proximal nerve trunks but can also involve the cranial nerves. CIDP is characterized by a broad spectrum of clinical phenotypes ( $20 \%$ atypical) [28]. The most frequent MR imaging features are bilateral, symmetric hypertrophy and hyperintensity of the BP and LSP nerve roots, with gradual normalization toward the distal segments [29] (Fig. 20.9). Patients with nerve root hypertrophy usually have a relapsing-remitting course and a longer disease duration, which may be related to demyelination and remyelination.

Hereditary neuropathies such as Charcot-Marie-Tooth disease can mimic the appearance of CIDP on MRI with findings of focal or diffuse peripheral nerve enlargement and fatty degeneration of the involved muscles. The crosssectional measurement of the sciatic nerve area in mid-thigh can be a helpful distinguishing feature [30].

Multifocal acquired demyelinating sensory and motor neuropathy (MADSAM) is characterized by an asymmetric multifocal pattern of motor and sensory loss, conduction block, and other features of demyelination in nerve conduction studies. Nerve hypertrophy is usually asymmetric and multifocal in the peripheral nerve trunks [31].

Multifocal motor neuropathy (MMN) is a chronic, slowly progressive immune-mediated neuropathy, characterized by progressive, predominantly distal, asymmetric limb weakness, mostly affecting upper limbs with minimal or no sensory impairment. The clinical presentation of MMN may mimic motor neuron disease, particularly in patients with predominant lower motor neuron impairment. MRI can be valuable to differentiate MMN from other neuropathies. About 40-50\% of the patients with MMN show asymmetric hypertrophy and signal intensity abnormalities or contrast enhancement on MRI, and the pattern of signal alterations closely correlates with the distribution of muscle weakness [32].

\subsection{Peripheral Nerve Tumors}

The most common neurogenic tumors of the BP and LSP are schwannomas and neurofibromas. On MRI, peripheral nerve sheath tumors are typically fusiform or round-shaped soft tissue lesions in continuity with the nerve and show classic findings such as the target, fascicular, and tail signs on T2 images, split fat sign on $\mathrm{T}$ images, and the bag of worms sign for plexiform neurofibromas [33]. Conventional MRI sequences cannot reliably differentiate solitary neurofibromas from schwannomas, although certain imaging features such as the surrounding T2 hypointense epineurium may be more typical of schwannomas than neurofibromas. 


\section{Key Point}

- When MRN shows diffuse T2 hyperintensity and enlargement of affected neural structures in a patient with acute onset including neck, shoulder, and scapula pain, look for the characteristic "bulls' eye sign" to suggest idiopathic brachial plexitis or idiopathic neuralgic amyotrophy.
A schwannoma arises from a single nerve fascicle, tends to be encapsulated, and grows eccentrically, compressing normal adjacent axons [34]. Multiplanar 3D MRN can accurately display this anatomical relationship of a schwannoma attached to the main trunk of a nerve. Schwannomas are iso- to hypointense on T1 images and hyperintense T2 images and demonstrate variable enhancement, though more homogeneous than neurofibromas (Fig. 20.10). Approximately $40 \%$ of schwannomas may demonstrate internal cystic change and $10 \%$ may show hemorrhage.
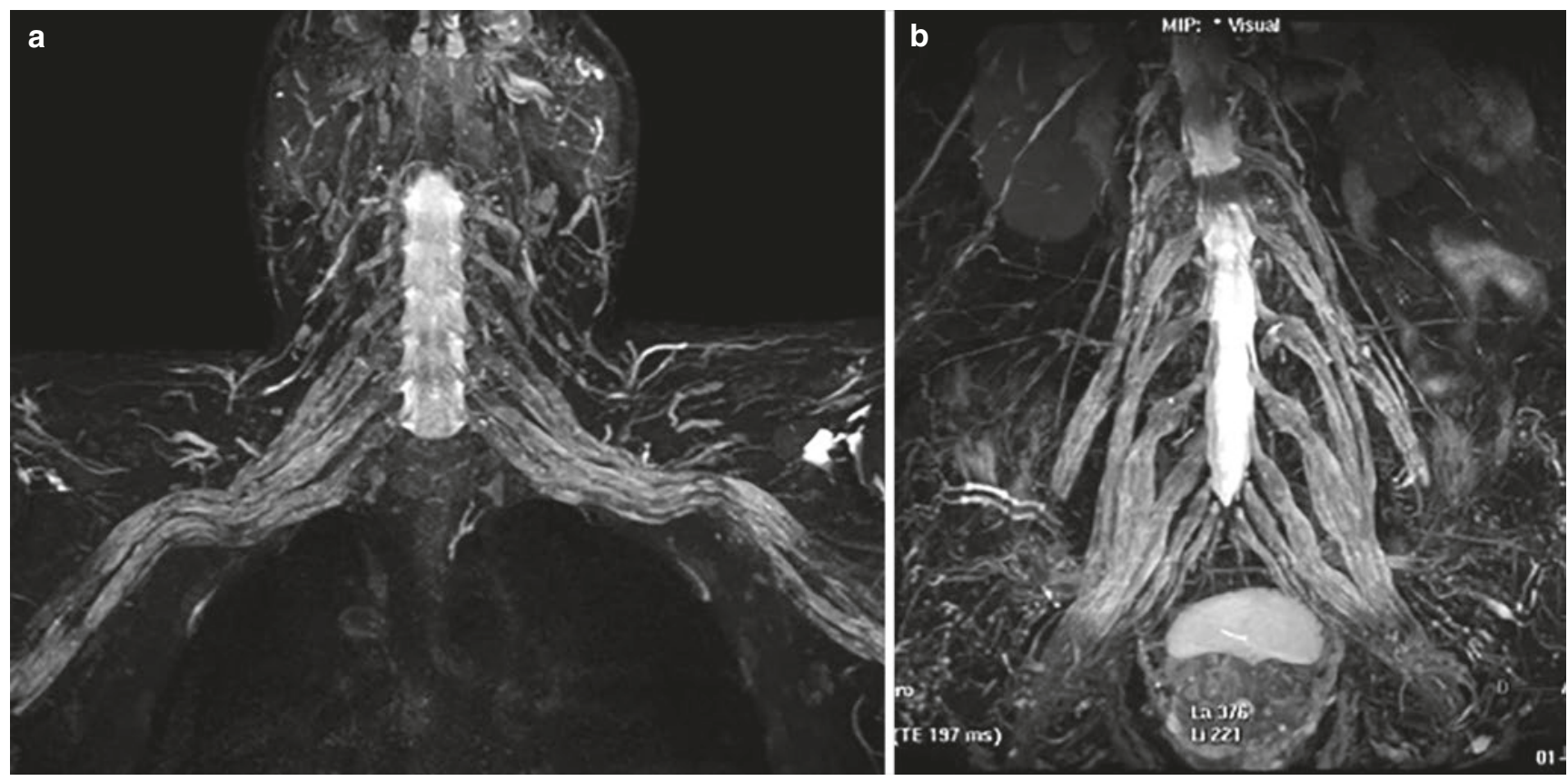

Fig. 20.9 50-year-old male, CIDP: 3D MRN, MIP views bilateral hypertrophy of the brachial (a) and lumbosacral plexus (b)
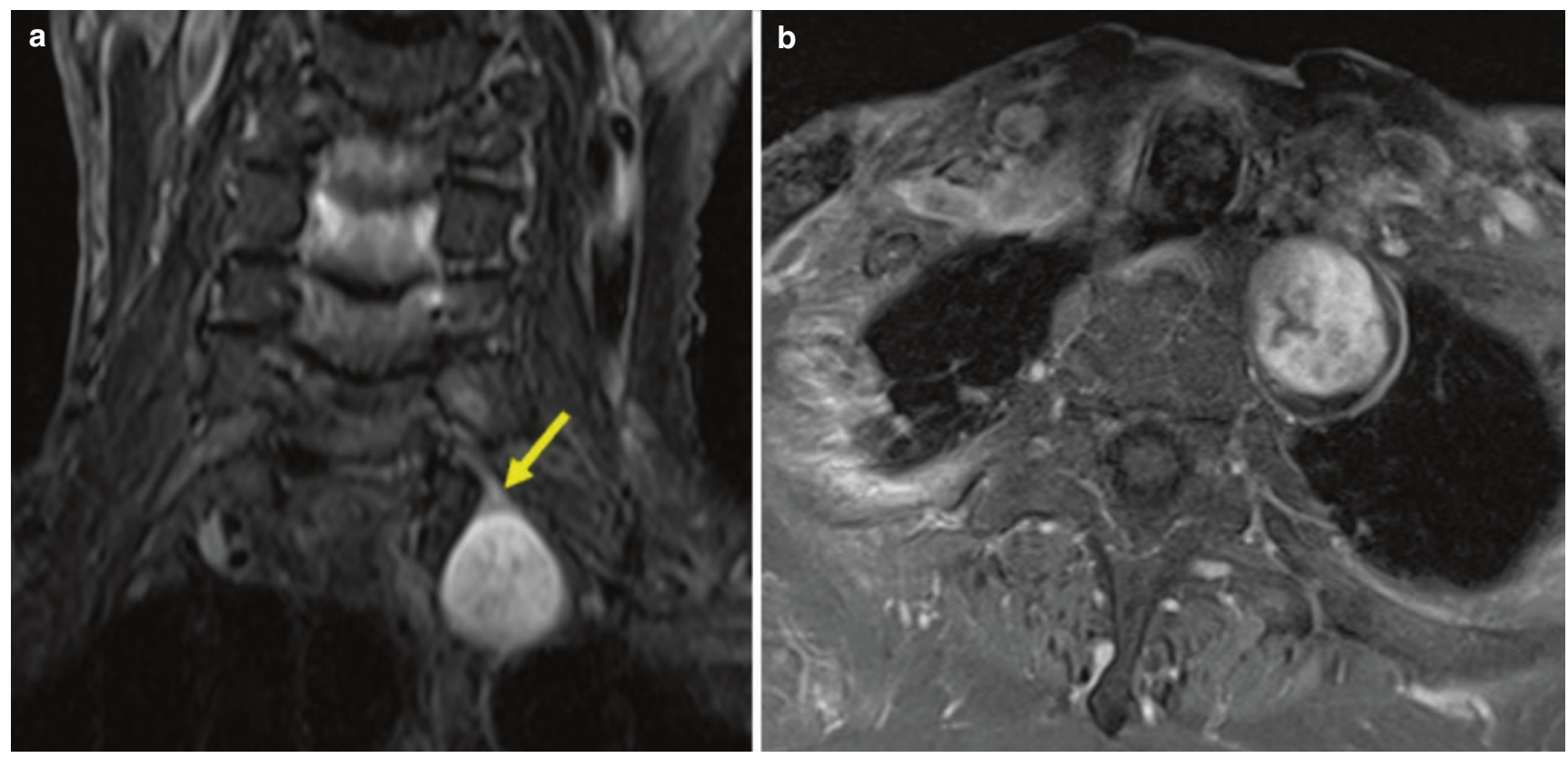

Fig. 20.10 (a) Coronal STIR and (b) axial contrast-enhanced T1 images of a schwannoma demonstrate the origin from a single nerve (arrow) and STIR hyperintensity. The heterogeneous enhancement may reflect internal cystic change 
A neurofibroma is composed of all peripheral nerve cellular elements (Schwann cells, fibroblasts, perineural cells, and axons), grows diffusely within and along nerves, and is not encapsulated. The nerves expand radially while entrapping native neural elements within the substance of the tumor. Approximately one-third of neurofibromas occur in the setting of neurocutaneous syndromes. Plexiform neurofibromas are pathognomonic for neurofibromatosis I (NF1). Neurofibromas are isointense on $\mathrm{T} 1$ images with high peripheral signal and low-to-intermediate signal on T2 images ("target sign"), reflecting the distribution of myxoid material and fibrous tissue (Fig. 20.11). They tend to show variable contrast enhancement. Multiplanar 3D MRN illustrates that neurofibromas are not separated from the normal nerve.

Malignant peripheral nerve sheath tumors (MPNST) have higher prevalence in patients with NF1 and can develop as a long-term side effect of radiation therapy. Malignant degeneration of plexiform neurofibromas can occur in 5-13\% of cases. The imaging distinction between benign nerve sheath tumors and MPNST can be challenging. Imaging features, such as advanced local invasion, bone destruction, poorly defined margins, absence of the target sign on T2 images, and larger size $(>5 \mathrm{~cm})$ suggest malignancy [34]. The combination of MRI findings (e.g., rapid growing, peripheral enhancement, peritumoral edema, intralesional cysts) has been reported to provide $61 \%$ sensitivity and $90 \%$ specificity in detecting MPNST [35]. ${ }^{18}$ Fluorodeoxyglucose positron emission tomography is a complementary diagnostic tool, particularly for identifying tumors with aggressive behavior [36].

Intraneural perineurioma is a rare benign and slowly growing nerve tumor arising from the perineurial cells surrounding the peripheral nerve fibers, most commonly occurring in teenagers and young adults without sex predilection and presenting with progressive muscle weakness.

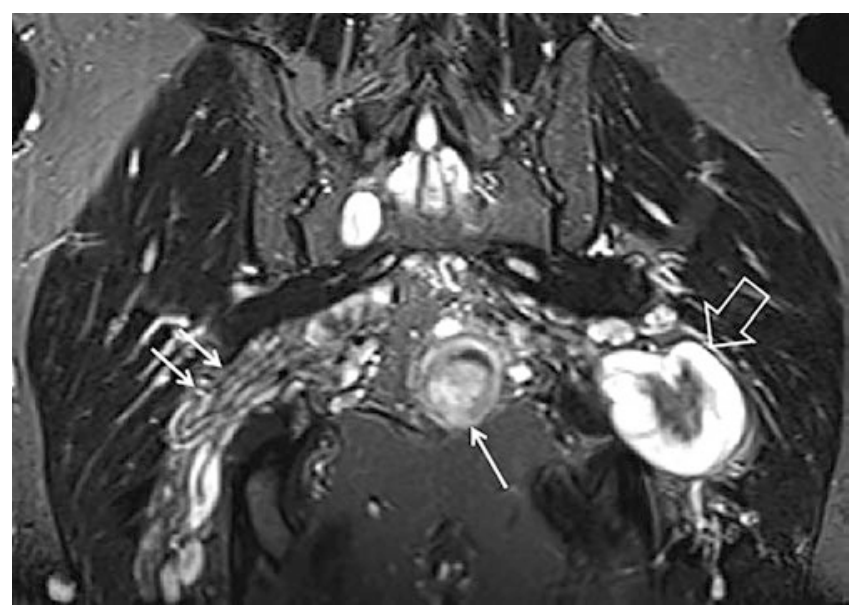

Fig. 20.11 Coronal MRN of a patient with NF1: neurofibroma of the left sciatic nerve with the "target" sign (empty arrow), neurofibroma with intermediate signal intensity of the pelvic floor (arrow), plexiform neurofibroma of the right sciatic nerve (double arrow)
Because of the insidious clinical presentation, the diagnosis is often delayed. Perineuriomas have typical MRI features: enlargement of the involved nerve over a considerable length, fusiform shape, mild increase in $\mathrm{T} 2$ signal intensity, T1 isointense signal, and moderate to marked contrast enhancement. Individual fascicles are uniformly enlarged, and their preserved fascicular architecture gives a "honeycomb" appearance on enhanced T1-fat-sat sequences.

Non-neurogenic tumors can cause plexopathy due to extrinsic compression or infiltration. Desmoid tumors, the most common non-neurogenic primary BP tumors, are isointense to muscle on $\mathrm{T} 1$ sequences and heterogeneously hyperintense on $\mathrm{T} 2$ sequences with areas of low $\mathrm{T} 2$ signal because of increased collagen content. They have infiltrative margins and show avid enhancement. Lipomas are characteristically isointense to fat on all MRI sequences and may externally compress BP and LSP. Lymphoma can encase the plexi due to enlarged lymph nodes and, when primarily involving the nerve roots and trunks, demonstrates increased signal and nerve enlargement. In neurolymphomatosis, there is neoplastic endoneurial invasion, most often associated with non-Hodgkin's lymphoma [37]. Prominent asymmetry, pain, and nodular MRI appearance may help distinguish neurolymphomatosis from paraneoplastic immune demyelinating radiculoneuropathy. MRN shows T2/STIR hyperintensity, focal and diffuse nerve enlargement with fascicular disorganization, and enhancement [37]. Primary neoplasms in close vicinity to the $\mathrm{BP}$, such as bronchogenic carcinoma/Pancoast tumors of the thoracic apex (Fig. 20.12); neurogenic tumors of the vagus nerve, phrenic nerve, and sympathetic trunk; metastatic lymph nodes, and bone tumors can cause nerve irritation/compression or direct invasion into the BP. A helpful clue to identify a Pancoast tumor is to look for intermediate signal effacing the normal $\mathrm{T} 1$ fat signal superior to the lung apex, medial to the scalene muscles, and lateral to the spine. Axial images are useful to demonstrate possible paraspinal extension of tumor in patients with complex symptoms, such as brachial plexopathy and Horner syndrome (second order neuron).

\section{Key Point}

- MRN can accurately display the anatomical relationship of a schwannoma attached to the main trunk of a nerve while a neurofibroma, composed of all peripheral nerve cellular elements, expands radially entrapping native neural elements within the tumor. A plexiform neurofibroma involves multiple nerve roots and has a "bag of worms" appearance. A perineurioma shows fusiform enlargement and mild T2 hyperintensity of the involved nerve and preserved fascicular architecture giving the "honeycomb" appearance on enhanced images. 

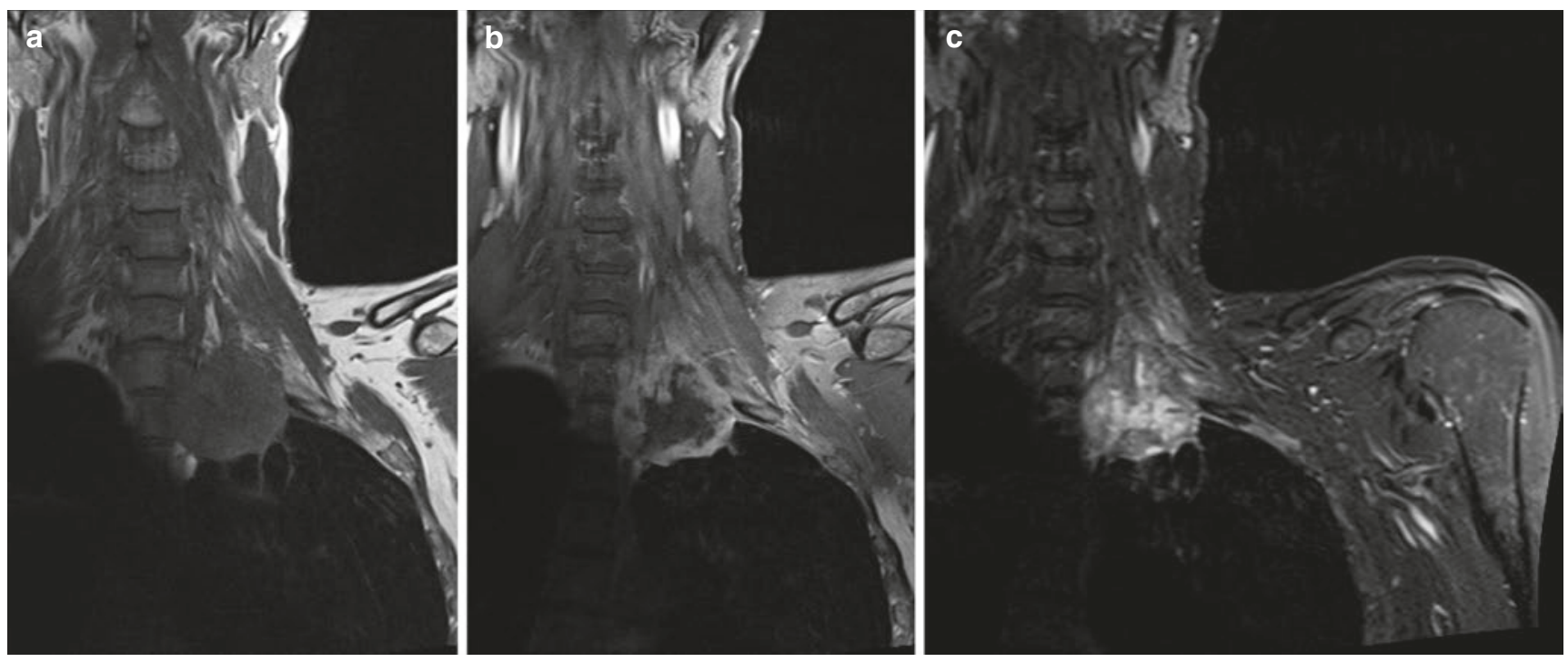

Fig. 20.12 Coronal (a) T1, (b) contrast-enhanced T1, and (c) STIR images reveal a $\mathrm{T} 1$ hypointense, heterogeneously $\mathrm{T} 2$ hyperintense, heterogeneously enhancing lesion in the left lung apex in keeping with a

Non-tumoral masses, such as aneurysms/pseudoaneurysms can cause a compressive plexopathy. DTT can provide relevant topographical information on non-neurogenic soft tissue tumors and adjacent nerves when conventional MRI is unable to depict the course of the involved nerves or distinguish possible nerve infiltration [38]. DTT and low FA provide insight into neural integrity, with low diffusivity values indicating malignancy [10]. As increasing ADC suggests benignity of lesions, this diffusion metric can also be potentially followed as a biomarker to detect tumor response/necrosis if the lesions are being followed longitudinally or after adjuvant medical treatment.

\subsection{Concluding Remarks}

Understanding of the brachial plexus and lumbosacral plexus anatomy is important for the accurate interpretation. Using an anatomic landmark-based approach to interrogate each individual component of the brachial plexus and lumbosacral plexus in combination with the direct and indirect imaging findings enables the development of a relevant differential diagnosis. By incorporating the clinical history, ancillary test results, and physical examination with the imaging findings, one can arrive at the correct diagnosis.

\section{Take Home Messages}

- Diagnostic work-up of peripheral neuropathies should include clinical history, physical examination, electrophysiological studies, and high resolution MRI/MRN (US when nerves are superficial). bronchogenic carcinoma (Pancoast tumor) infiltrating into the interscalene triangle and involving the left $\mathrm{C} 7, \mathrm{C} 8$, and T1 (arrow). This patient presented with persistent left shoulder pain
- MRN affords the advantage of simultaneous exploration of nerves, muscles, and other surrounding tissues which can assess secondary effects of nerve pathology (e.g., muscle denervation), and compressive or infiltrative pathologies.

- It is important to distinguish between preganglionic and postganglionic traumatic plexopathy as it affects treatment options, with the former requiring nerve transfer and the latter often being managed conservatively.

- In peripheral nerve entrapment syndromes, US has the advantage of providing dynamic evaluation of nerve entrapment. MRN is able to identify a variable degree of signal intensity change within the nerve that is commonly higher proximal to the site of entrapment.

- Neurogenic tumors are identified as well-circumscribed, round-shaped soft tissue lesions in continuity with the nerve, and MRI findings of advanced local invasion, poorly defined margins, absence of the target sign on T2-weighted sequences, peritumoral edema, intralesional cysts, and bone destruction, suggest malignancy.

\section{References}

1. Pham M, Baumer T, Bendszus M. Peripheral nerves and plexus: imaging by MR-neurography and high-resolution ultrasound. Curr Opin Neurol. 2014;27(4):370-9.

2. Padua L, Liotta G, Di PA, Granata G, Pazzaglia C, Caliandro P, et al. Contribution of ultrasound in the assessment of nerve diseases. Eur J Neurol. 2012;19(1):47-54. 
3. Rubin JA, Wesolowski JR. Neck MR imaging anatomy. Magn Reson Imaging Clin N Am. 2011;19(3):457-73.

4. Moore KR, Tsuruda JS, Dailey AT. The value of MR neurography for evaluating extraspinal neuropathic leg pain: a pictorial essay. AJNR Am J Neuroradiol. 2001;22(4):786-94.

5. Filler AG, Maravilla KR, Tsuruda JS. MR neurography and muscle MR imaging for image diagnosis of disorders affecting the peripheral nerves and musculature. Neurol Clin. 2004;22(3):643.

6. Aagaard BD, Maravilla KR, Kliot M. Magnetic resonance neurography: magnetic resonance imaging of peripheral nerves. Neuroimaging Clin N Am. 2001;11(1):viii, 131-46.

7. Bendszus M, Koltzenburg M, Wessig C, Solymosi L. Sequential MR imaging of denervated muscle: experimental study. AJNR Am J Neuroradiol. 2002;23(8):1427-31.

8. Chhabra A, Ahlawat S, Belzberg A, Andreseik G. Peripheral nerve injury grading simplified on MR neurography: as referenced to Seddon and Sunderland classifications. Indian J Radiol Imaging. 2014;24(3):217-24.

9. Dong Q, Jacobson JA, Jamadar DA, Gandikota G, Brandon C, Morag $\mathrm{Y}$, et al. Entrapment neuropathies in the upper and lower limbs: anatomy and MRI features. Radiol Res Pract. 2012;2012:230679.

10. Ahlawat S, Chhabra A, Blakely J. Magnetic resonance neurography of peripheral nerve tumors and tumorlike conditions. Neuroimaging Clin N Am. 2014;24(1):171-92.

11. Chazen JL, Cornman-Homonoff J, Zhao Y, Sein M, Feuer N. MR neurography of the lumbosacral plexus for lower extremity radiculopathy: frequency of findings, characteristics of abnormal intraneural signal, and correlation with electromyography. AJNR Am J Neuroradiol. 2018;39(11):2154-60.

12. Trivedi JR, Phillips L, Chhabra A. Hereditary and acquired polyneuropathy conditions of the peripheral nerves: clinical considerations and MR neurography imaging. Semin Musculoskelet Radiol. 2015;19(2):130-6.

13. Jeon T, Fung MM, Koch KM, Tan ET, Sneag DB. Peripheral nerve diffusion tensor imaging: overview, pitfalls, and future directions. J Magn Reson Imaging. 2018;47(5):1171-89.

14. Tagliafico A, Calabrese M, Puntoni M, Pace D, Baio G, Neumaier $\mathrm{CE}$, et al. Brachial plexus MR imaging: accuracy and reproducibility of DTI-derived measurements and fibre tractography at 3.0T. Eur Radiol. 2011;21(8):1764-71.

15. Chhabra ATS, Andreisek G. Nerve anatomy, pathophysiology, nerve injury and entrapment. In: Chhabra AAG, editor. Editor. Magnetic resonance neurography. New Delhi: JayPee Brothers Medical Publishers; 2012. p. 1-9.

16. Doi K, Otsuka K, Okamoto Y, Fujii H, Hattori Y, Baliarsing AS. Cervical nerve root avulsion in brachial plexus injuries: magnetic resonance imaging classification and comparison with myelography and computerized tomography myelography. J Neurosurg. 2002;96(3 Suppl):277-84.

17. Yoshikawa T, Hayashi N, Yamamoto S, Tajiri Y, Yoshioka N, Masumoto T, et al. Brachial plexus injury: clinical manifestations, conventional imaging findings, and the latest imaging techniques. Radiographics. 2006;26(Suppl 1):S133-43.

18. Gasparotti R, Lodoli G, Meoded A, Carletti F, Garozzo D, Ferraresi S. Feasibility of diffusion tensor tractography of brachial plexus injuries at $1.5 \mathrm{~T}$. Investig Radiol. 2013;48(2):104-12.

19. Simon NG, Narvid J, Cage T, Banerjee S, Ralph JW, Engstrom JW, et al. Visualizing axon regeneration after peripheral nerve injury with magnetic resonance tractography. Neurology. 2014;83(15):1382-4.

20. Lang EM, Borges J, Carlstedt T. Surgical treatment of lumbosacral plexus injuries. J Neurosurg Spine. 2004;1(1):64-71.

21. Jarvik JG, Comstock BA, Heagerty PJ, Haynor DR, Fulton-Kehoe $\mathrm{D}$, Kliot $\mathrm{M}$, et al. Magnetic resonance imaging compared with electrodiagnostic studies in patients with suspected carpal tunnel syndrome: predicting symptoms, function, and surgical benefit at 1 year. J Neurosurg. 2008;108(3):541-50.
22. Wang H, Ma J, Zhao L, Wang Y, Jia X. Utility of MRI diffusion tensor imaging in carpal tunnel syndrome: a meta-analysis. Med Sci Monit. 2016;22:736-42.

23. Sneag DB, Saltzman EB, Meister DW, Feinberg JH, Lee SK, Wolfe SW. MRI Bullseye sign: an indicator of peripheral nerve constriction in parsonage-turner syndrome. Muscle Nerve. 2017;56(1): 99-106.

24. van Eijk J, Chan YC, Russell JW. Immunotherapy for idiopathic lumbosacral plexopathy. Cochrane Database Syst Rev. 2013;12:CD009722. https://doi.org/10.1002/14651858.CD009722. pub2.

25. Qayyum A, MacVicar AD, Padhani AR, Revell P, Husband JE. Symptomatic brachial plexopathy following treatment for breast cancer: utility of MR imaging with surface-coil techniques. Radiology. 2000;214(3):837-42.

26. Aralasmak A, Cevikol C, Karaali K, Senol U, Sharifov R, Kilicarslan R, et al. MRI findings in thoracic outlet syndrome. Skelet Radiol. 2012;41(11):1365-74.

27. Byun WM, Park WK, Park BH, Ahn SH, Hwang MS, Chang JC. Guillain-Barre syndrome: MR imaging findings of the spine in eight patients. Radiology. 1998;208(1):137-41.

28. Mathey EK, Park SB, Hughes RA, Pollard JD, Armati PJ, Barnett $\mathrm{MH}$, et al. Chronic inflammatory demyelinating polyradiculoneuropathy: from pathology to phenotype. J Neurol Neurosurg Psychiatry. 2015;86(9):973-85.

29. Van den Bergh PY, Hadden RD, Bouche P, Cornblath DR, Hahn A, Illa I, et al. European Federation of Neurological Societies/ Peripheral Nerve Society guideline on management of chronic inflammatory demyelinating polyradiculoneuropathy: report of a joint task force of the European Federation of Neurological Societies and the Peripheral Nerve Society - first revision. Eur J Neurol. 2010;17(3):356-63.

30. Sinclair CD, Miranda MA, Cowley P, Morrow JM, Davagnanam I, Mehta $\mathrm{H}$, et al. MRI shows increased sciatic nerve cross sectional area in inherited and inflammatory neuropathies. J Neurol Neurosurg Psychiatry. 2011;82(11):1283-6.

31. Rajabally YA, Chavada G. Lewis-sumner syndrome of pure upperlimb onset: diagnostic, prognostic, and therapeutic features. Muscle Nerve. 2009;39(2):206-20.

32. Van Asseldonk JT, Van den Berg LH, Van den Berg-Vos RM, Wieneke GH, Wokke JH, Franssen H. Demyelination and axonal loss in multifocal motor neuropathy: distribution and relation to weakness. Brain. 2003;126(Pt 1):186-98.

33. Thawait SK, Chaudhry V, Thawait GK, Wang KC, Belzberg A, Carrino JA, et al. High-resolution MR neurography of diffuse peripheral nerve lesions. AJNR Am J Neuroradiol. 2011;32(8):1365-72.

34. van Es HW, Bollen TL, van Heesewijk HP. MRI of the brachial plexus: a pictorial review. Eur J Radiol. 2010;74(2):391-402.

35. Matsumine A, Kusuzaki K, Nakamura T, Nakazora S, Niimi R, Matsubara $\mathrm{T}$, et al. Differentiation between neurofibromas and malignant peripheral nerve sheath tumors in neurofibromatosis 1 evaluated by MRI. J Cancer Res Clin Oncol. 2009;135(7): 891-900.

36. Broski SM, Johnson GB, Howe BM, Nathan MA, Wenger DE, Spinner RJ, et al. Evaluation of (18)F-FDG PET and MRI in differentiating benign and malignant peripheral nerve sheath tumors. Skelet Radiol. 2016;45(8):1097-105.

37. Bourque PR, Warman Chardon J, Bryanton M, Toupin M, Burns BF, Torres C. Neurolymphomatosis of the brachial plexus and its branches: case series and literature review. Can J Neurol Sci. 2018;45(2):137-43.

38. Kasprian G, Amann G, Panotopoulos J, Schmidt M, Dominkus M, Trattnig S, et al. Peripheral nerve tractography in soft tissue tumors: a preliminary 3-tesla diffusion tensor magnetic resonance imaging study. Muscle Nerve. 2015;51(3):338-45. 
Open Access This chapter is licensed under the terms of the Creative Commons Attribution 4.0 International License (http://creativecommons. org/licenses/by/4.0/), which permits use, sharing, adaptation, distribution and reproduction in any medium or format, as long as you give appropriate credit to the original author(s) and the source, provide a link to the Creative Commons license and indicate if changes were made.

The images or other third party material in this chapter are included in the chapter's Creative Commons license, unless indicated otherwise in a credit line to the material. If material is not included in the chapter's Creative Commons license and your intended use is not permitted by statutory regulation or exceeds the permitted use, you will need to obtain permission directly from the copyright holder.

(c) (1) 